\title{
Algorithms for the m-Coverage Problem and k-Connected m-Coverage Problem in Wireless Sensor Networks
}

\author{
Deying $\mathrm{Li}^{1,2}$, Jiannong $\mathrm{Cao}^{3}$, Dongsheng Liu ${ }^{1}$, Ying $\mathrm{Yu}^{4}$, and Hui Sun ${ }^{1}$ \\ ${ }^{1}$ School of Information, Renmin University of China, Beijing, China, 100872 \\ ${ }^{2}$ Key Laboratory of Data Engineering and Knowledge Engineering, MOE \\ ${ }^{3}$ Department of Computing, The Hong Kong Polytechnic University, HongKong \\ ${ }^{4}$ School of Information Engineering, University of Science and Technology of Beijing
}

\begin{abstract}
An important issue in deploying a wireless sensor network (WSN) is to provide target coverage with high energy efficiency and fault-tolerance. In this paper, we study the problem of constructing energy-efficient and faulttolerant target coverage with the minimal number of active nodes which form an $m$-coverage for targets and a $k$-connected communication subgraph. We propose two heuristic algorithms for $m$-coverage problem, and get the performance ratio of one heuristic. Then two heuristic algorithms are further proposed to solve the $k$-connected $m$-coverage problem. The simulation results demonstrate the desired efficiency of the proposed algorithms.
\end{abstract}

Keywords: $k$-connected $m$-coverage, sensor networks, approximation algorithm, energy-efficient.

\section{Introduction}

Monitoring a geographical region or a set of targets and collecting the relevant data are very important tasks in wireless sensor networks. Since sensor nodes are often deployed in an arbitrary manner, one of the fundamental issues in the task of target monitoring is target coverage which reflects how well the deployed sensor nodes can monitor a set of targets. Meanwhile, the energy-efficiency is another important issue in WSNs. In general, sensor nodes are powered by very limited battery resources. Recent research has found that significant energy savings can be achieved by elaborate managing the duty cycle of nodes in WSNs with high node density. In this approach, some nodes are scheduled to sleep (or enter a power saving mode) while the remaining active nodes keep working.

Sensing is only one responsibility of a sensor network. To operate successfully, most sensor networks must also remain connected, i.e., the active nodes should not be partitioned in any configured schedule of node duty cycles. A sensor network must provide satisfactory connectivity so that nodes can communicate for data fusion and reporting the results to base stations. Single connectivity often is not sufficient for many sensor networks because a single failure could disconnect the network, and single coverage is also not sufficient. Therefore, maintaining sufficient sensing 
coverage and network connectivity with minimal active nodes are critical requirements in WSNs.

In this paper, we study more general coverage problem-- $k$-connected $m$-coverage problem: to find minimized number of active nodes to form a target $m$-coverage and meanwhile any pair of active nodes is connected by at least $k$ disjoint paths. To solve the $k$-connected $\mathrm{m}$-coverage problem, we first investigate an introductory problem, namely $m$-coverage problem that is to find the minimum number of active nodes ensuring that each target can be covered by at lease $m$ distinct sensor nodes. We show that the $m$-coverage problem is NP-hard and then give one heuristic and an approximation algorithm accordingly. Next, based on the $k$-connected coverage problem [19], we propose two heuristic algorithms to solve the $k$-connected $m$ coverage problem.

The rest of the paper is organized as follows. In section 2 we present related works. Section 3 describes network model and problems studied in this paper. Section 4 and 5 propose two heuristics for $m$-coverage and $k$-connected $m$-coverage problem respectively. Section 6 describes the simulations and section 7 concludes the paper.

\section{Related Works}

There are many studies on the coverage problem ([1-5 etc.]) in WSNs. Different formulations of the coverage problem have been proposed, depending on the subject to be covered (area versus discrete points) [4,5], the sensor deployment mechanism (random versus deterministic [6]), as well as other wireless sensor network properties (e.g. network connectivity and minimum energy consumption). For energy efficient area coverage, the works in [7] and [8] consider a large population of sensors, deployed randomly for area monitoring.

Zhang and Hou [9] prove an important, but intuitive result that if the communication range $\mathrm{Rc}$ is at least twice the sensing range Rs, a complete coverage of a convex area implies connectivity of the working nodes. They further discuss the case of Rc $>$ Rs. Wang et al [10] generalize the result in [9]. Wu and Yang [11] propose two density control models for energy conserving protocol in sensor networks, using the adjustable sensing range of several levels. Zhou et al $[12,13]$ address the problem of selecting a minimum size connected k-cover.

The energy-efficient target coverage problem deals with the problem of covering a set of targets with minimum energy cost [1,6,14]. Cardei and Du [1] address the target coverage problem where the disjoint sets are modeled as disjoint set covers, such that every cover completely monitors all the target points. Cardei et. [14] propose an approach different from [1] by not requiring the sensor sets to be disjoint and by allowing sensors to participate in multiple sets, and design two heuristics that efficiently compute the sets, using linear programming and a greedy approach.

Alam[15] et al consider coverage and connectivity in 3-Dimensional networks. Liu $[16,17,18]$ et al consider maximal lifetime scheduling for sensor surveillance systems with $k$ sensors to 1 target. In these papers, they assume each sensor watch at most a target and each target is watched by at least $k$ sensor. 
In [19] we addressed the $k$-connected coverage problem for targets. In this paper we extend our work [19] to $k$-connected $m$-coverage problem. Our model is different from [16-18], in our model, a sensor may watch all targets in its sensing range.

\section{Network Model and Problem Specification}

In this section, we formulate the target $m$-coverage problem and the $k$-connected $m$ coverage problem addressed in this paper.

Let us assume that $n$ sensors $v_{1}, v_{2}, \ldots . . v_{n}$ are deployed in a region to monitor $t$ targets $I_{1}, I_{2}, \ldots I_{t}$. Each node $v_{i}$ has a sensing region $S\left(v_{i}\right)$ and communication range $R$. Any target inside $S\left(v_{i}\right)$ is cover by $v_{i}$. $v_{i}$ can directly communicate with $v_{j}$ if their Euclidian distance is less than communication range $R$. Consequently, the sensor nodes in the communication network can form a undirected graph $G=(V, E)$, where $V=\left\{v_{1}, v_{2}, \ldots v_{n}\right\}$ is a set of sensor nodes and $E$ is a set of edges $(i, j)$. Without loss of generality, assume $T=\left\{I_{1}, I_{2}, \ldots I_{t}\right\}$ to be a given set of targets. For each sensor $v \in V$, there is a subset $T_{v}$ of $T=\left\{I_{1}, I_{2}, \ldots I_{t}\right\}$, which is covered by $v$. Note that the targets are different from the sensor nodes.

The graph is $k$-connected if there are $k$ node-disjoint paths between any pair of nodes. A set of sensors $C \subseteq V$ is said to be $m$-coverage if each target in $T$ is covered by at least $m$ distinct sensor nodes in $C$.

In order to reduce the energy consumption, our work is to minimize the number of sensor nodes.

Thus, the problem studied in this paper can be now formally defined as follows:

$\boldsymbol{m}$-coverage problem: Given a graph $G=(V, E)$ and a set of targets $T$, we want to find a minimal number of sensor nodes in $V$, where these nodes form a $m$-coverage for targets.

$\boldsymbol{k}$-connected $\boldsymbol{m}$-coverage problem: Given a graph $G=(V, E)$ and a set of targets $T$, we want to find a minimal number of sensor nodes in $V$, where these nodes form a $m$ coverage for targets and the subgraph induced by these nodes is $k$-connected.

\section{Approximation Algorithms to m-Coverage Problem}

In this section, we will first investigate an introductory problem, namely $m$-coverage problem, of the $k$-connected $m$-coverage problem. This problem is NP-hard as it is a generalization of set cover, which is already known to be NP-hard. We present two heuristics for $m$-coverage problem. We first model the $m$-coverage problem as Integer Programming in section 4-A, and then use the relaxation technique to design a linear programming based heuristic in section 4-B. Next, we propose a heuristic based on duality rounding and give its approximation ratio in section $4-\mathrm{C}$. 


\section{A. Integer programming Formulation of the m-coverage problem}

We formulate the $m$-coverage problem as follows:

\section{Given:}

$n$ : The total number of sensor nodes; $t$ : The total number of targets;

$j$ : Indicator for sensor nodes, $j \in[1, n] ; i$ : Indicator for targets, $i \in[1, t]$. $a_{i j}= \begin{cases}1 & \text { if target } i \text { is covered by sensor } j ; \\ 0 & \text { otherwise }\end{cases}$

Variable:

$x_{j}= \begin{cases}1 & \text { if } j \text { is selected for } m \text { - coverage; } \\ 0 & \text { otherwise }\end{cases}$

The $m$-coverage problem then can be formulated as 0-1 programming as follows:

ILP:

$$
Z_{1}=\operatorname{Min} \sum_{j=1}^{n} x_{j}
$$

subject to:

$$
\begin{aligned}
& \sum_{j=1}^{n} a_{i j} x_{j} \geq m \quad i \in[1, t] \\
& x_{j}=0 \text { or } 1 \quad j \in[1, n]
\end{aligned}
$$

\section{B. LP heuristic}

This heuristic is a two-stage algorithm. At the first stage, an optimal solution for a linear programming (LP) relaxation of the ILP is computed. The obtained solution to LP may be fractional, so it may not satisfy the integer constraint (3). At the second stage, a greedy algorithm is employed to find an integral solution based on the optimal solution obtained at the first stage.

\section{LP Heuristic:}

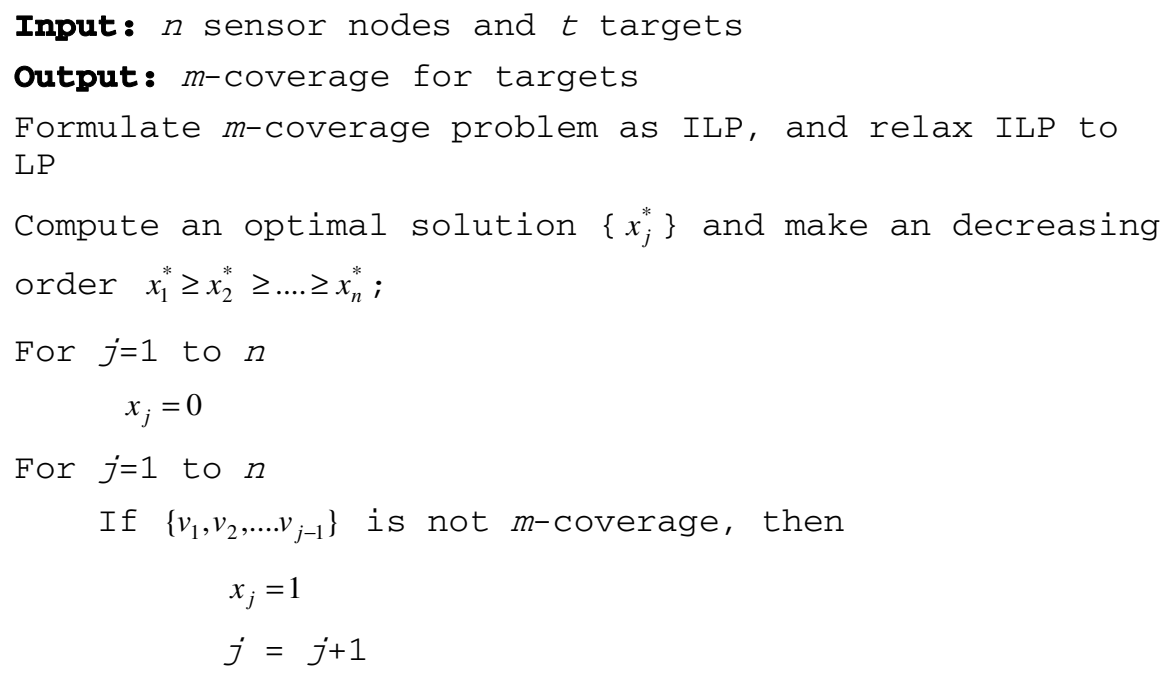


We have seen that in the above heuristic, the optimal solution to a linear programming relaxation is employed to find out the priority of variables being assigned with 1 . There is a disadvantage with this approach: Computing the optimal solution takes $O\left(n^{3.5}\right)$ time for LP of $n$ variables [20]. It is the main portion of the total computation time for this heuristic. In order to reduce the computational time and improve the quality of output solution, we will design another heuristic by applying rounding by duality.

\section{Heuristic based on rounding by duality}

To simplify the description of this heuristic, we consider the primal form of linear programming (PLP).

\section{PLP}

$$
Z_{2}=\operatorname{Min} \sum_{j=1}^{n} c_{j} x_{j}
$$

subject to

$$
\begin{aligned}
& \sum_{j=1}^{n} a_{i j} x_{j} \geq b_{i} \quad i \in[1, t] \\
& 0 \leq x_{j} \leq 1 \quad j \in[1, n]
\end{aligned}
$$

Where $a_{i j}$ is 1 or $0, b=(m, m, \ldots ., m)^{T}$ in which there are $t$ 's $m . c=(1,1, \ldots ., 1)$ in which there is $n$ 's $1 . x^{T}=\left(x_{1}, x_{2}, \ldots, x_{n}\right)$ is variable vector. The dual of the above linear programming is DLP, and $y^{T}=\left(y_{1}, y_{2}, \ldots, y_{t}\right), z^{T}=\left(z_{1}, z_{2}, \ldots ., z_{n}\right)$ are dual variable vector.

We use a two-stage algorithm to find an approximation solution for it. First, a feasible solution is obtained for the Dual Linear Programming problem (DLP). The corresponding solution for the Primal form (PLP) is obtained by the rounding procedure:

The formal description of the algorithm is given below:

\section{Heuristic based on rounding by duality}

Initially, set $x^{0}=0, \quad\left(y^{0}, z^{0}\right)=(0,0), \quad k=0 / / x^{0}, y^{0}, z^{0}$
are vectors

While $x^{k}$ is not prime feasible do begin

$$
\begin{aligned}
& \text { Set } J(k)=\left\{j \mid x_{j}^{k}=0\right\} ; \\
& \text { Set } I(k)=\left\{i \mid \sum_{j=1}^{n} a_{i j} x_{j}^{k} \leq b_{i}-1\right\} ; \\
& \text { Choose } \quad r \in J(k) \text { such that } \\
& \frac{c_{r}-\sum_{i=1}^{t} a_{i r} y_{i}^{r}}{\sum_{i \in I(k)} a_{i r}}=\alpha=\min _{j \in J}\left\{\frac{c_{j}-\sum_{i=1}^{t} a_{i j} y_{i}}{\sum_{i \in I(k)} a_{i j}} \mid c_{j}-\sum_{i=1}^{t} a_{i j} y_{i} \geq 0\right\}
\end{aligned}
$$




$$
\begin{aligned}
& \text { Set } x_{j}^{k+1}=x_{j}^{k} \text { if } j \neq r \text { and } x_{r}^{k+1}=1 ; \\
& \text { Set } y_{i}^{k+1}=y_{i}^{k}+\alpha \text { if } i \in I(k) \text { and } y_{i}^{k+1}=y_{i}^{k} \text { if } i \notin I(k) \\
& \text { Set } z_{j}^{k+1}=\max \left(\sum_{i=1}^{t} a_{i j} y_{i}^{k+1}-c_{j}, 0\right) ; \\
& k \leftarrow k+1 ;
\end{aligned}
$$

\section{end-while}

Output $x^{k}$ with $Z_{1 A}=\sum_{j=1}^{n} c_{j} x_{j}^{k}$

Theorem. The performance ratio of heuristic based on rounding by duality is $f=\max _{1 \leq i \leq t} \sum_{j=1}^{n} a_{i j}$, and the time complexity is $O\left(n^{2}\right)$.

\section{Approximation Algorithm for $k$-Connected $m$-Coverage Problem}

In this section, we address the $k$-connected $m$-coverage problem which is NP-hard because $m$-coverage problem is NP-hard. We will design two heuristic algorithms. One is called as $k m \mathrm{TS}$ algorithm, the other is called $k m$ Reverse algorithm.

A. kmTS algorithm: The main idea of $k m$ TS algorithm is that the algorithm includes two steps: the first step is to construct a $m$-coverage of targets; The second step is to increase small size nodes to this $m$-coverage such that the subgraph by these increased nodes and nodes of $m$-coverage is $k$-connected. For the first step, we may use the algorithms in section 4 to get an approximation for $m$-coverage problem. For the second step, we may use our algorithms [19] to get solution for $k$-connected $m$ coverage problem.

kmTS Algorithm: Construct an approximate solution for $k$-connected $m$-coverage

Input: Given $G=(V, E)$, a set $T$ of targets, and

$T_{v}, \forall v \in V$, which is a subset of $T$ covered by $V$

Output: $k$-connected $m$-coverage for $T$

(1) Construct $m$-coverage $C$ for $T$ using $m$-coverage heuristic.

(2) Connect set $C$ into $k$-connected subgraph, i.e.

finding a subset $X$ of $V-C$ to $C$ such that $G[C \cup X]$ is $k-$ connected subgraph and $|X|$ is minimized.

B. kmReverse algorithm: In the following, we will give another algorithm--reverse algorithm which directly apply Lemma[19]. The main idea of kmReverse algorithm is as follows: initially, each sensor node in the sensor network is active, then, change one active node to inactive node each time if it satisfies two conditions (1) deleting 
the node, the remain nodes also form a $m$-coverage (2) any two neighbours of the node has $k$-node disjoint paths in remain graph after deleting the node.

kmReverse algorithm: Construct an approximate solution

Input: Given $G=(V, E)$, a set $T$ of targets, and $T_{v}, \forall v \in V$, which is a subset of $T$ covered by $V$.

Output: $k$-connected $m$-coverage for $T$

1. $V^{k}:=V$;

2. Sort all nodes in $V$ in an increasing order of degree in $T$ as $v_{1}, v_{2}, \ldots v_{n}$ such that $D_{T}\left(v_{1}\right) \leq D_{T}\left(v_{2}\right) \leq \ldots \leq D_{T}\left(v_{n}\right)$, where $D_{T}(v)=\left\{r_{j} \mid r_{j}\right.$ is covered by $\left.v\right\} \mid$

3. For $i=1$ to $n$,

$$
\begin{aligned}
& \text { if } \forall u_{1}, u_{2} \in N\left(v_{i}\right), u_{1} \text { is } k \text {-connected to } u_{2} \text { in } G\left[V^{k}-\left\{v_{i}\right\}\right], \\
& \text { and } V^{k}-\left\{v_{i}\right\} \text { is a m-coverage for } T, \\
& \qquad V^{k}=V^{k}-\left\{v_{i}\right\} \\
& \qquad i:=i+1
\end{aligned}
$$

\section{Performance Evaluation}

In this section we evaluate the performance of proposed algorithms. We simulate a stationary network with sensor nodes and target points randomly located in a $500 \times 500$ area. We assume the sensing range is equal for all the sensors in the network, and the communicating range is also equal for all the sensors in the network. In the simulation we consider the following tunable parameters:

- $N$, the number of sensor nodes, which varies between 40 and 80 .

- $M$, the number of targets to be covered, which varies between 10 and 26.

- $R$, the communicating range which varies between 120 to 200 .

- $S$, the sensing range, which varies between 70 and 110 .

The simulation is conducted in a $500 \times 5002$-D free-space by independently and uniformly allocating $N$ nodes and $M$ targets. All nodes have the same transmission range $R$. And all nodes have the same sensing range $S$. For any pair of nodes, if the distance between the two nodes is no more than the value of transmission range $R$, there exists an edge between the two nodes. For any sensor node and any target, if the distance between the sensor and the target is no more than the value of sensing range, the target is covered by the sensor node. We present averages of 100 separate runs for each result shown in figures. In each run of the simulations, for given $N$ and $M$, we randomly place $N$ nodes in the square, and randomly place $M$ nodes as targets. Any topology which is not connected or targets are not covered by all sensor nodes is discarded. 


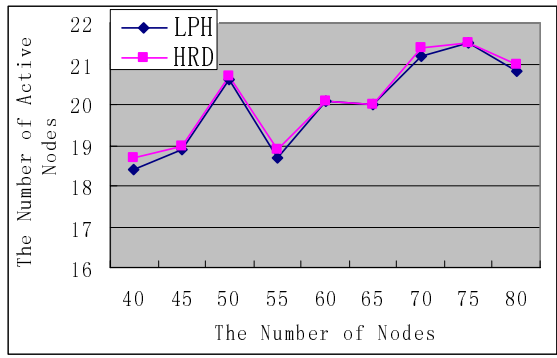

(a) $m=3, M=10, S=70$

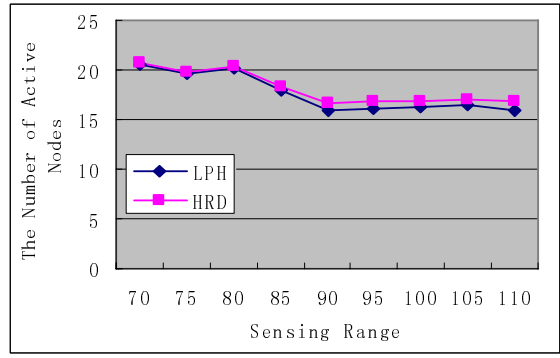

(b) $m=3, M=10, N=50$

Fig. 1. The number of active nodes with the number of nodes, sensing range

In the first experiment, we simulate the proposed LP Heuristic (LPH) and Heuristic based on rounding by duality(HRD) for $m$-coverage problem. The results show the performances of the two heuristics are close from Fig.1 and Fig. 2.

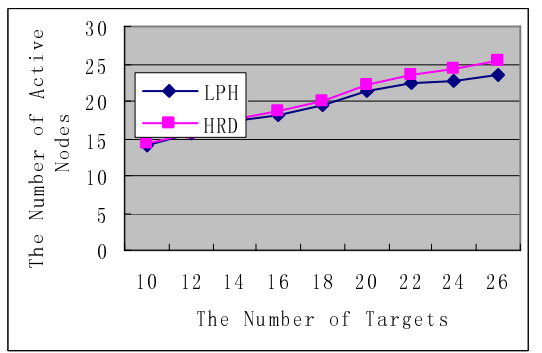

(a) $m=2, S=70, N=70$

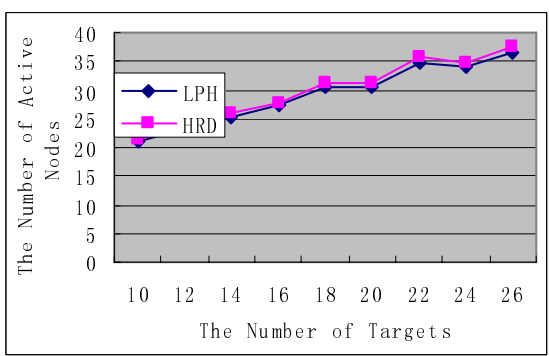

(b) $m=3, S=70, N=70$

Fig. 2. The number of active nodes with number of targets

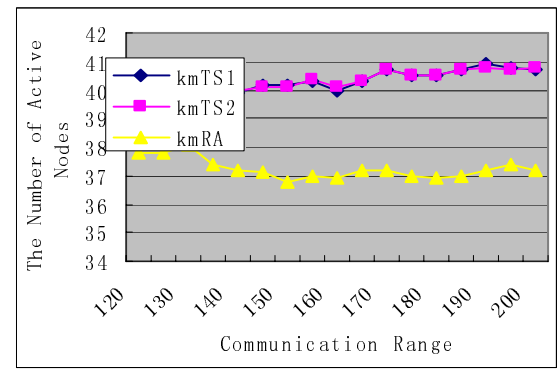

(a) $k=3, m=3, M=10, N=50, S=70$

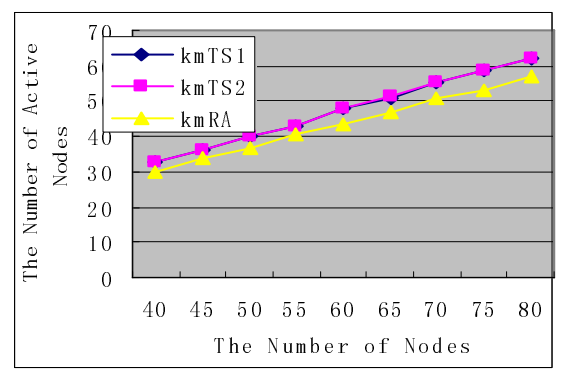

(b) $k=3, m=3, M=10, R=150, S=70$

Fig. 3. The number of active nodes with comm. range, the number of nodes 


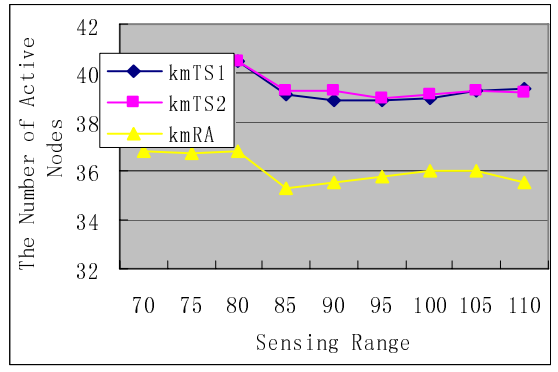

(a) $k=3, m=3, M=10, R=150, N=50$

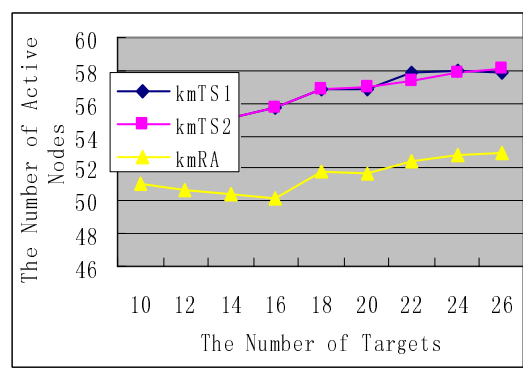

(b) $k=3, m=3, N=70, R=150, S=70$

Fig. 4. The number of active nodes with sensing range, the number of targets

In the second experiment, we simulate the proposed kmTS and kmRA and compare their performances. We call $\mathrm{kmTS}_{1}$ and $\mathrm{kmTS}_{2}$ when kmTS using LPH and HRD respectively. The simulation results are shown in Fig.3 and Fig.4. The number of active nodes increases with the number of sensors and the number of targets, as more sensors need to participate so that each active pairs communicate with k-disjoint paths and more targets needs to be covered. The number of active sensors is not increased with increasing sensing range, because when sensing range is larger each target is covered by more sensors.

\section{Conclusions}

In this paper, we study how to construct $k$-connected $m$-coverage with minimized number of active sensors for targets in wireless sensor networks. We first discuss the $m$-coverage problem in WSNs, we propose two heuristics and get performance ration of one. Then based on the discussion of the $m$-coverage and [19], we propose two heuristics to construct $k$-connected $m$-coverage. We also carry out extensive simulations for our algorithms and the obtained simulation results have demonstrated the high effectiveness of our algorithms.

Acknowledgement. This research is partially supported by the National Natural Science Foundation of China under grant 10671208.

\section{References}

1. Cardei, M., Du, D.Z.: Improving Wireless Sensor Network Lifetime through Power Aware Organization. ACM Wireless Networks (to appear)

2. Cardei, M., MarCallum, D., Cheng, X., Min, X., Jia, X., Li, D.Y., Du, D.-Z.: Wireless Sensor Networks with Energy Efficient Organization. Journal of Interconnection Networks 3(3-4), 213-229 (2002)

3. Cardei, M., Wu, J.: Energy-Efficient Coverage Problems in Wireless Ad Hoc Sensor Networks. Journal of Computer Communications on Sensor Networks (to appear) 
4. Li, X., Wan, P., Wang, Y., Frieder, O.: Coverage in Wireless Ad-hoc Sensor Networks. IEEE Transactions on Computers 52(6), 753-763 (2003)

5. Meguerdichian, S., Koushanfar, F., Potkonjak, M., Srivastava, M.B.: Coverage Problems in Wireless Ad-hoc Sensor Networks. In: Proc. of INFOCOM'01, pp. 1380-1387 (2001)

6. Kar, K., Banerjee, S.: Node Placement for Connected Coverage in Sensor Networks. In: Proc. of WiOpt 2003 (2003)

7. Slijepcevic, S., Potkonjak, M.: Power Efficient Organization of Wireless Sensor Networks. In: Proc. of IEEE International Conference on Communications, vol. 2, pp. 472-476 (2001)

8. Huang, C., Tseng, Y.: The Coverage Problem in a Wireless Sensor Network. In: WSNA'03, September 19, 2003, San Diego, California, USA (2003)

9. Zhang, H., Hou, J.C.: Maintaining Sensing Coverage and Connectivity in Large Sensor Networks. In: NSF International workshop on Theoretical and algorithmic Aspects of sensor, Ad Hoc Wireless and Peer-to-Peer Networks (February 2004)

10. Wang, X., Xing, G., Zhang, Y., Lu, C., Pless, V., Gill, C.D.: Integrated Coverage and Connectivity Configuration in Wireless Sensor Networks. In: Proc. of the First ACM Conference on Embedded Networked Sensor Systems, ACM Press, New York (2003)

11. Wu, J., Yang, S.: Energy-Efficient Node Scheduling Models in Sensor Networks with Adjustable Ranges. International Journal of Foundations of Computer Science (February 2005)

12. Zhou, Z., Das, S., Gupta, H.: Connected K-Coverage Problem in Sensor Networks. In: ICCCN 2004, pp. 373-378 (2004)

13. Zhou, Z., Das, S., Gupta, H.: Fault Tolerant Connected Sensor Cover with Variable Sensing and Transmission Ranges. In: IEEE SECON 2005 (2005)

14. Cardei, M., Thai, M., Li, Y., Wu, W.: Energy-Efficient Traget Coverage in Wireless Sensor Networks. In: INFOCOM 2005 (2005)

15. Alam, S.: Coverage and connectivity in Three-Dimensional Networks. In: MobiCom'06, pp. 346-357 (2006)

16. Liu, H., Wan, P., Jia, X., Makki, S., Pissinou, N.: Maximal Lifetime Scheduling in Sensor Surveillance Networks. In: IEEE INFOCOM'05, Miami (March 2005)

17. Liu, H., Wan, P., Jia, X.: Maximal Lifetime Scheduling for Sensor Surveillance Systems with K Sensors to 1 Target. IEEE Trans on Parallel and Distributed Systems 17(12), 15261536 (2006)

18. Liu, H., Jia, X., Wan, P., Yi, C., Makki, S., Pissinou, N.: Maximizing Lifetime of Sensor Surveillance Systems. IEEE/ACM Transactions on Networking (Accepted)

19. Li, D., Cao, J., Liu, M., Zheng, Y.: K-connected Coverage Problem in Wireless Sensor Networks. In: COCOA2007 (2007)

20. Schrijver, A.: Theory of linear and integer programming (1985) 